\title{
Regulation of thalamic development by Sonic hedgehog
}

\author{
Douglas J. Epstein* \\ Department of Genetics, Perelman School of Medicine, University of Pennsylvania, Philadelphia, PA, USA
}

\section{Edited by:}

Steffen Scholpp, Karlsruhe Institute of

Technology, Germany

\section{Reviewed by:}

James Y.H Li, University of

Connecticut Health Center, USA

Clemens Martin Kiecker, King's

College London, UK

*Correspondence:

Douglas J. Epstein, Department of

Genetics, Perelman School of

Medicine, University of Pennsy/vania,

Clinical Research Building, Room 470,

415 Curie Blvd, Philadelphia, PA

19104, USA.

e-mail: epsteind@mail.med.

upenn.edu
The thalamus is strategically positioned within the caudal diencephalic area of the forebrain, between the mesencephalon and telencephalon. This location is important for unique aspects of thalamic function, to process and relay sensory and motor information to and from the cerebral cortex. How the thalamus comes to reside within this region of the central nervous system has been the subject of much investigation. Extracellular signals secreted from key locations both extrinsic and intrinsic to the thalamic primordium have recently been identified and shown to play important roles in the growth, regionalization, and specification of thalamic progenitors. One factor in particular, the secreted morphogen Sonic hedgehog (Shh), has been implicated in spatiotemporal and threshold models of thalamic development that differ from other areas of the CNS due, in large part, to its expression within two signaling centers, the basal plate and the zona limitans intrathalamica, a dorsally projecting spike that separates the thalamus from the subthalamic region. Shh signaling from these dual sources exhibit unique and overlapping functions in the control of thalamic progenitor identity and nuclei specification. This review will highlight recent advances in our understanding of Shh function during thalamic development, revealing similarities, and differences that exist between species.

\section{THE PROSOMERE MODEL OF FOREBRAIN DEVELOPMENT}

Almost 20 years ago, Puelles and Rubenstein (1993) described a model to help explain how the complex architecture of the mouse forebrain is generated from discrete developmental territories termed prosomeres. The purpose of the prosomere model was to relate the bent longitudinal axis of the forebrain to that of more posterior regions of the neural tube and to define its primary subdivisions along the anteroposterior $(\mathrm{a} / \mathrm{p})$ and dorsoventral axes. Initially, the spatial patterns of 45 genes were mapped onto the prosomeric model with many respecting the hypothesized transverse and longitudinal boundaries of the forebrain (Puelles and Rubenstein, 1993). Over the years, hundreds of new genes have been added to the list and further testing of the model has led to its reinterpretation (Puelles and Rubenstein, 2003). As it stands, the prosomere model stipulates that the caudal forebrain is organized into three prosomeres $(\mathrm{p} 1-\mathrm{p} 3)$ corresponding to the pretectum, thalamus, and prethalamus, respectively, whereas the rostral forebrain (telencephalon and hypothalamus) represents a complex protosegment not divided into prosomeres (Figure 1).

Functional genetic experiments performed over the past decade in several model organisms have further validated the prosomere model and have greatly enhanced our understanding of the molecular mechanisms underlying forebrain formation and evolution (Hébert and Fishell, 2008; Scholpp and Lumsden, 2010). A common theme that has emerged from these and other studies of nervous system development is that compartmentalization of the neuroepithelium into functional units is facilitated by its exposure to extrinsic factors secreted from localized signaling centers (Jessell, 2000; Hébert and Fishell, 2008; Scholpp and Lumsden, 2010). In the case of the caudal forebrain, the secreted morphogen Sonic hedgehog (Shh), has been shown to play a multifaceted role in regulating the growth and identity of distinct neuronal progenitor subtypes within the thalamic complex, as well as the formation of the zona limitans intrathalamica (zli), a dorsally projecting boundary between $\mathrm{p} 2$ and $\mathrm{p} 3$ that also serves as a critical signaling center for thalamic and prethalamic development (Figure 1). This review will highlight the diverse functions of Shh at different stages of thalamic development, including a feature unique to the caudal forebrain whereby Shh secreted from two orthogonal sources (basal plate and zli) contributes to a morphogenic signaling gradient that patterns an alar structure, the thalamic primordium.

\section{Shh SIGNALING IN THE SPINAL CORD: LESSONS LEARNED FROM 20 YEARS OF STUDY}

Much of what we know about Shh signaling has come from studies of its role in spinal cord development. A summary of the principal concepts learned from this work is described below and will serve as a framework for comparison with the roles of Shh signaling during thalamic development. For more comprehensive reviews on this subject the reader is encouraged to consult the following references (Dessaud et al., 2008; Matise and Wang, 2011).

Sonic hedgehog is a secreted protein that provides positional information to a wide variety of developing tissues, including the CNS (Dessaud et al., 2008; Ingham et al., 2011; Matise and Wang, 2011). Shh is expressed in the axial mesoderm (prechordal plate and notochord) and ventral midline (floor plate) of the overlying neural tube throughout most of the a/p neuraxis (Echelard et al., 1993; Roelink et al., 1994). It is from these sources that a ventral to dorsal concentration gradient of Shh is established in the ventral neural tube. Over the past several years a compelling body of evidence has been generated to explain how the Shh signaling gradient 


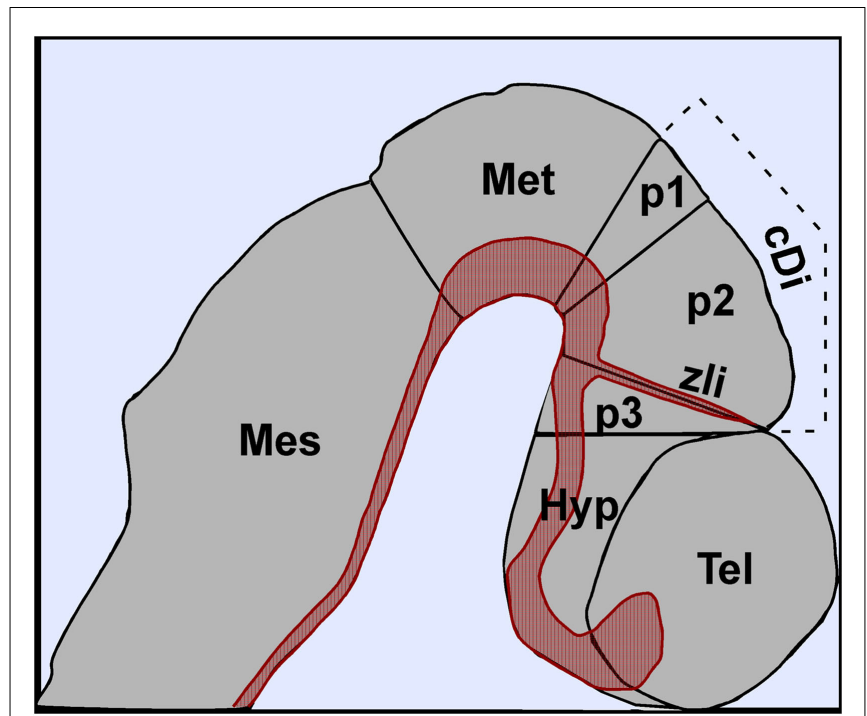

FIGURE 1 | Regionalization of the central nervous system along the anteroposterior axis of the mouse at embryonic day 10.5. The caudal diencephalon is divided into three prosomeres (p1, p2, p3) corresponding to the pretectum, thalamaus, and prethalamus, respectively. The zona limitans intrathalamica (zli) is located at the p2-p3 boundary. The expression of Shh in the ventral midline and zli is depicted in red. Abbreviations: cDi, caudal diencephalon; Hyp, hypothalamus; Mes, mesencephalon; Met, metencephalon; Tel, telencephalon. length Gli proteins and their transcriptional activation of target genes, including Gli1 and Ptch1.

In response to its position along the Shh morphogen gradient, a progenitor cell elicits distinct temporal profiles of Gli activity (Stamataki et al., 2005; Dessaud et al., 2007, 2010). This is a dynamic process given that progenitors become desensitized to Shh over time, as a result of the negative feedback loop with Ptch1. Therefore, to keep its position along the dorsoventral axis, the progenitor cell must maintain a certain threshold of Shh signaling over time (Dessaud et al., 2010).

Each progenitor domain can be identified by the expression of a distinct set of homeodomain and bHLH transcription factors (Briscoe and Ericson, 2001; Lupo et al., 2006). Boundaries between progenitor domains are generated over time by the mutual repression of complementary pairs of transcription factors (Muhr et al., 2001). Once the boundaries are fixed, the unique combination of transcription factors assigned to a given progenitor domain further directs the fate of differentiating neurons.

\section{THE MULTIPLE ROLES OF Shh DURING THALAMIC DEVELOPMENT}

While significant advances have been made in elucidating the requirements of Shh signaling in posterior regions of the CNS, it is only recently that similar progress has been described for the diencephalon (Scholpp and Lumsden, 2010). The thalamic primordium develops from the alar plate of $\mathrm{p} 2$. Shh expression is localized to the basal plate of p1-p3 by the 12-somite stage of development and over a day later (25-somites) is initiated in the zli, where it becomes fully activated by E10.5 (Figure 1).

The exposure of the thalamus to two Shh signaling centers has made it somewhat of a challenge to reconcile the specific roles of either one in regulating the growth, patterning and neuronal identity of thalamic progenitors. However, recent studies using a combination of genetic and tissue perturbation approaches in mouse, chicken, and zebrafish embryos have developed a clearer picture of the multifaceted roles of Shh signaling during thalamic development (Hashimoto-Torii et al., 2003; Kiecker and Lumsden, 2004; Vieira et al., 2005; Scholpp et al., 2006; Szabó et al., 2009; Vue et al., 2009; Jeong et al., 2011).

\section{EARLY ROLES FOR Shh AS A MITOGEN}

The dependency of thalamic development on Shh is temporally regulated. As early as the 15-somite stage of mouse development, $\mathrm{Shh}^{-/-}$embryos show reduced proliferation and survival of diencephalic precursors (Ishibashi and McMahon, 2002). This mitogenic role for Shh occurs well before zli formation and is therefore attributed to Shh signaling from the prechordal plate and/or ventral midline of the diencephalon. Since the cell proliferation defects in $\mathrm{Sh} \mathrm{h}^{-1-}$ embryos were also observed in alar regions of the diencephalon, well out of range of Shh secreted from ventral sources, a Shh-dependent relay signal was proposed to regulate the growth of thalamic progenitors. Fgf15 appeared to be an ideal candidate to fulfill this function as its dorsal growth promoting properties were dependent on Shh, at least when overexpressed in cultured mouse brain explants (Ishibashi and McMahon, 2002). However, recent loss of function studies do not support this conclusion, as $\mathrm{Fg} f 5^{-/-}$ mutants show increased proliferation of dorsal neural progenitors 
and decreased neurogenesis in the developing midbrain and neocortex, consistent with Fgf15 functioning as a growth suppressor (Borello et al., 2008; Fischer et al., 2011). The identity of the Shhdependent regulator of thalamic growth and survival remains to be identified. Wnt ligands are good candidates based on the finding that several are expressed in the thalamic primordium, as well as the fact that the expression of $T c f 4$, a transcriptional mediator of Wnt signaling, is downregulated in the diencephalon of $\mathrm{Shh}^{-1-}$ embryos (Ishibashi and McMahon, 2002). While Wnt signaling may, in some instances, act antagonistically to Shh in the specification of some neural cell fates (Robertson et al., 2004), this is likely to be context dependent, as Wnt signaling is also dependent on Shh for the proliferation of neural progenitors in the spinal cord (Alvarez-Medina et al., 2009).

\section{Zli FORMATION}

The epichordal/prechordal interface marks the territory from where the zli will emerge (Vieira et al., 2005). The expression of the homeodomain transcription factor Otx2 on the posterior (epichordal) side of the zli and the zinc finger proteins Fezf1 and Fezf2 on the anterior (prechordal) side of the $\mathrm{p} 2 / \mathrm{p} 3$ border are required for zli formation (Hirata et al., 2006; Jeong et al., 2007; Scholpp et al., 2007). Whether these transcription factors play a direct role in regulating $S h h$ expression in the zli, or provide a permissive environment for Shh to be transcribed, remains unresolved. Interestingly, mouse mutants lacking the bHLH trancription factors Hes 1 and Hes5 also show a loss of Shh expression in the zli (Baek et al., 2006). It is intriguing to speculate that Hes1/5 might be functioning downstream of a cross repressive interaction between Otx 2 and Fezf2 to regulate Shh expression in the zli. Of course, other regulatory relationships are equally possible.

The zli is a key partition between the thalamic and prethalamic territories and also serves as an important signaling center for the regionalization of the $a / p$ axis of the caudal diencephalon (Scholpp and Lumsden, 2010). The zli extends dorsally from the ventral midline at the $\mathrm{p} 2 / \mathrm{p} 3$ boundary, coinciding with the anterior limit of the notochord (Figure 1; Vieira et al., 2005). Shh expression is first detected in the zli at the 25 somite stage of chick and mouse embryos and expands dorsally at a rate of $\sim 20 \mu \mathrm{m} / \mathrm{h}$ until it reaches a length of $600 \mu \mathrm{m}$ (Zeltser, 2005). The dorsal progression of Shh transcription was inhibited in chick embryos upon insertion of a microbarrier between the basal plate and the zli, or when Shh signaling was blocked with a constitutively active form of Ptch1 (Kiecker and Lumsden, 2004; Zeltser, 2005; Vieira and Martinez, 2006). These results suggested that Shh expression in the zli is regulated by a ligand-dependent feed-forward signaling mechanism.

A Shh-dependent vertical signaling model to explain the spread of Shh transcription along the zli is not entirely consistent with the finding that Shh continues to be expressed in the zli of mutant mouse embryos lacking principle components of the Shh transduction cascade, including Gli2, Gli3, and Smo (Hashimoto-Torii et al., 2003; Vue et al., 2009). Since it is likely that the inactivation of Smo function in Nestin-cre; Smo loxp/loxp embryos occurred after Shh expression was already initiated in the zli, these results might suggest that the maintenance, but not the initiation, of Shh expression in the zli is independent of Shh signaling. A more substantial challenge to the vertical signaling model comes from the observation that $S h h$ expression was initiated in the zli of Gli2 $^{-1-}$; Gli3 $^{-/-}$mouse mutants, as well as zebrafish oep mutants (which lack the nodal co-receptor tdgf1/cripto), despite the lack of Shh expression in the basal plate of the caudal diencephalon, which was thought to be the initiating source of the vertical signal (Hashimoto-Torii et al., 2003; Scholpp et al., 2006). Clearly, more work will be needed to sort out the molecular details of zli formation. A more thorough analysis of the critical cis and trans determinants of Shh expression in the zli may help explain the direct regulatory mechanisms underlying the formation of this structure (Epstein et al., 1999; Jeong et al., 2006).

The thalamus and prethalamus express different sets of genes in response to Shh signaling from the zli (Kiecker and Lumsden, 2004; Scholpp et al., 2006; Vieira and Martinez, 2006). To explain how this differential response to Shh is orchestrated, Kiecker and Lumsden (2004) proposed that the thalamus and prethalamus are prepatterned. In support of their hypothesis, they showed that the homeobox gene Irx3 acts as a thalamic competence factor. When misexpressed in the prethalamus of chick embryos, Irx 3 ectopically activated genes typically expressed posterior to the zli in a Shh-dependent manner. While loss of function studies with Irx3 are likely confounded by functional redundancy with other family members, it is nonetheless intriguing that in zebrafish Irx $1 \mathrm{~b}$ morphants, the zli is posteriorly expanded at the expense of the thalamus, suggesting that Irxlb is necessary to restrict zli formation on the epichordal side of the zli (Scholpp et al., 2007).

\section{Shh PATTERNS THE THALAMUS ALONG A MORPHOGENIC GRADIENT}

The spatial arrangement of thalamic nuclei is important for generating the precise topographical relationship needed to fulfill its role as a relay center. Despite the many advances in our knowledge of the early events regulating thalamic growth, and regionalization, we still know relatively little concerning the mechanisms by which heterogeneous clusters of thalamic neurons become specified and aggregate into discrete thalamic nuclei. One particular challenge has been to correlate the patterns of gene expression initiated by Shh and other signaling pathways at early stages of thalamic development with discrete nuclei and/or neuronal subtypes that form at later postnatal stages (Nakagawa and O'Leary, 2001; Jones and Rubenstein, 2004; Vue et al., 2007, 2009; Szabó et al., 2009; Suzuki-Hirano et al., 2011; Yuge et al., 2011).

The neurons contributing to thalamic nuclei are derived from at least two distinct progenitor domains. The caudal population of thalamic progenitors, $\mathrm{pTH}-\mathrm{C}$, is the larger of the two groups, and gives rise to all thalamic nuclei that relay sensory information from the periphery to primary sensory regions of the neocortex via thalamocortical axons (Figure 2; Vue et al., 2007). The rostral population of thalamic progenitors, $\mathrm{pTH}-\mathrm{R}$, comprises a narrow band of cells sandwiched between pTH-C and the zli (Figure 2). Thalamic neurons derived from pTH-R progenitors are thought to contribute to two dorsolaterally positioned thalamic nuclei, the ventrolateral geniculate nucleus (vLG), and the 


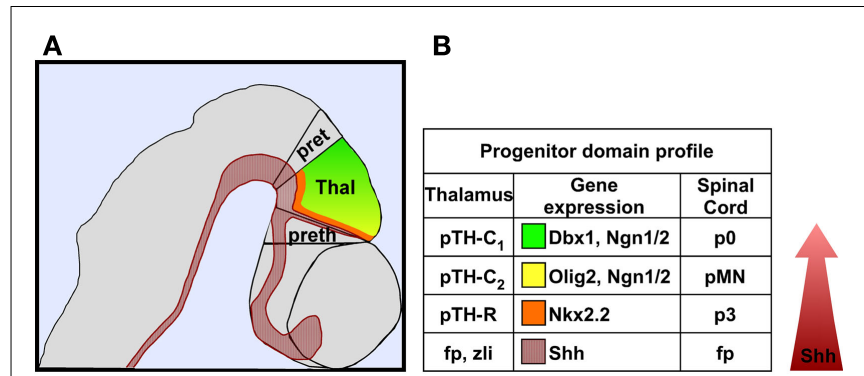

FIGURE 2 | A morphogenetic gradient of Shh signaling establishes distinct thalamic progenitor domains. (A) Schematic representation of a lateral view of the caudal diencephalon color-coded as follows: Shh expressing cells (red); $\mathrm{pTH}-\mathrm{C}_{2}$ domain (yellow); $\mathrm{pTH}-\mathrm{C}_{1}$ domain (green); pTH-R domain (orange). (B) Comparison of a representative set of genes expressed in thalamic and ventral spinal cord progenitor domains.

intergeniculate leaflet (IGL), neither of which project axons to the cortex (Horowitz et al., 2004; Morin and Blanchard, 2005; Jones, 2007; Vue et al., 2007, 2009).

In addition to its role in early patterning events, Shh signaling is also required to specify neuronal subtypes that contribute to a broad array of thalamic nuclei. The current model proposes that graded Shh signaling is necessary and sufficient to promote distinct classes of thalamic progenitors (Hashimoto-Torii et al., 2003; Scholpp et al., 2009; Szabó et al., 2009; Vue et al., 2009). The pTH-R domain, which develops closest to the zli, is dependent on the highest concentration of Shh, whereas, the rostroventral $\left(\mathrm{pTH}-\mathrm{C}_{2}\right)$ and caudodorsal $\left(\mathrm{pTH}-\mathrm{C}_{1}\right)$ populations of pTH-C progenitors, developing several cell diameters away from the zli, are dependent on progressively lower concentrations of Shh (Figure 2; Hashimoto-Torii et al., 2003; Szabó et al., 2009; Vue et al., 2009).

The conditional inactivation of either Shh or Smo in the diencephalon results in the loss of pTH-R progenitors and their post-mitotic derivatives in the vLG and IGL, as well as a sizeable reduction in the population of pTH-C progenitors and the Gbx2 expressing, cortex-projecting, thalamic neurons that differentiate from these cells (Szabó et al., 2009; Vue et al., 2009; Jeong et al., 2011). Although Szabó et al. (2009) and Vue et al. (2009) both described varying degrees of thalamic deficits in the absence of Shh, the phenotypes described by Szabó et al. (2009) were more severe and correlated with a greater loss of Gbx2 expression, which was likely attributed to the use of an earlier acting and more robust Cre line (Foxb1-Cre versus Netrin-Cre) to delete Shh from the thalamic primordium.

How does Shh signaling determine the different classes of thalamic progenitors? At first glance, a model similar to that described for neuronal subtype identity in the ventral spinal cord could be envisioned, whereby distinct thalamic progenitors are specified by their exposure to different thresholds of Shh signaling activity. However, another possibility is that the two classes of Shhdependent thalamic progenitors, $\mathrm{pTH}-\mathrm{R}$ and $\mathrm{pTH}-\mathrm{C}$, are specified by two spatially distinct sources of Shh, the basal plate, and zli, respectively. For the latter model to be valid, different phenotypes should arise from the inactivation of Shh from discrete signaling territories.

To help resolve this question, Jeong et al. (2011) examined mutant mice containing a targeted deletion of a Shh regulatory element required for Shh expression in the basal plate of the caudal diencephalon, but not the zli. This analysis showed that the expression of high threshold target genes in pTH-R (Nkx2.2, Ascl1, Tal1) was reduced, concomitant with an expanded expression domain of lower threshold, pTH-C target genes (Ngn2). While this result may, in part, reflect temporal differences in the dependency of pTH-R on Shh, it might also be the case that prolonged Shh signaling activity from both diencephalic sources is required to promote pTH-R identity. Given that the blockade of Shh signaling from the zli in chick embryos also results in a loss of $N k \times 2.2$ expression in the pTH-R domain, the most parsimonious explanation of the data is that both sources of Shh surrounding the thalamus are necessary for pTH-R identity (Kiecker and Lumsden, 2004; Jeong et al., 2011). Therefore, Shh secreted from two signaling sources, the basal plate and zli, supplies the Shh signaling gradient that shapes thalamic progenitor identity over time.

The similarity in signaling mechanisms by which Shh regulates neuronal progenitor subtype identity in the thalamus and ventral spinal cord also extends to the use of some of the same transcriptional regulators mediating these cell fate decisions (Figure 2). For instance, $\mathrm{pTH}-\mathrm{R}$ and $\mathrm{p} 3$ neuronal progenitors form closest to their respective sources of Shh in the thalamus and spinal cord, respectively, and both populations express Nkx2.2. The rostroventral pTH-C (classified as $\mathrm{pTH}-\mathrm{C}_{2}$ ) and $\mathrm{pMN}$ domains reside at slightly greater distances from their respective sources of Shh and both express the bHLH transcription factors Olig2, Ngn1, and Ngn2. Finally, the caudodorsal region of pTH-C in the thalamus (classified as pTH- $\mathrm{C}_{1}$ ) and $\mathrm{p} 0$ domain of the spinal cord both express Dbxl and are at the tail end of the Shh responsive territories.

\section{FUTURE DIRECTIONS}

The specificity of thalamic progenitors are not solely determined by Shh. Clearly, additional signaling pathways (Fgf, Wnt, and others) must play significant roles in generating the diversity of progenitor subtypes that have been, and remain to be, discovered in the thalamus (Braun et al., 2003; Zhou et al., 2004; Miyake et al., 2005; Vieira and Martinez, 2005; Kataoka and Shimogori, 2008; Bluske et al., 2009; Martinez-Ferre and Martinez, 2009; Peukert et al., 2011). Future research will undoubtedly uncover how these signaling pathways integrate to generate the transcriptional network that programs each of the thalamic progenitor domains and gives rise to the full complement of thalamic nuclei. Hopefully, in the not too distant future, our understanding of thalamic development will match that of the intricately detailed patterning events that occur in the spinal cord (Alaynick et al., 2011).

\section{ACKNOWLEDGMENTS}

Work in Douglas J. Epstein's lab is supported, in part, by a grant from the NIH/NINDS (R01 NS39421). 


\section{REFERENCES}

Alaynick, W. A., Jessell, T. M., and Pfaff, S. L. (2011). SnapShot: spinal cord development. Cell 146, 178-178.e1.

Allen, B. L., Song, J. Y., Izzi, L., Althaus, I. W., Kang, J. S., Charron, F., Krauss, R. S., and McMahon, A. P. (2011). Overlapping roles and collective requirement for the coreceptors GAS1, CDO, and BOC in SHH pathway function. Dev. Cell 20, 775-787.

Alvarez-Medina, R., Le Dreau, G., Ros, M., and Martí, E. (2009). Hedgehog activation is required upstream of Wnt signalling to control neural progenitor proliferation. Development 136, 3301-3309.

Baek, J. H., Hatakeyama, J., Sakamoto, S., Ohtsuka, T., and Kageyama, R. (2006). Persistent, and high levels of Hes1 expression regulate boundary formation in the developing central nervous system. Development 133, 2467-2476.

Bluske, K. K., Kawakami, Y., KoyanoNakagawa, N., and Nakagawa, Y. (2009). Differential activity of Wnt/beta-catenin signaling in the embryonic mouse thalamus. Dev. Dyn. 238, 3297-3309.

Borello, U., Cobos, I., Long, J. E., McWhirter, J. R., Murre, C., and Rubenstein, J. L. (2008). FGF15 promotes neurogenesis and opposes FGF8 function during neocortical development. Neural Dev. 3, 17.

Braun, M. M., Etheridge, A., Bernard, A., Robertson, C. P., and Roelink, H. (2003). Wnt signaling is required at distinct stages of development for the induction of the posterior forebrain. Development 130, 5579-5587.

Briscoe, J., and Ericson, J. (2001). Specification of neuronal fates in the ventral neural tube. Curr. Opin. Neurobiol. 11, 43-49.

Chen, Y., and Struhl, G. (1996). Dual roles for patched in sequestering and transducing Hedgehog. Cell 87, 553-563.

Dessaud, E., McMahon, A. P., and Briscoe, J. (2008). Pattern formation in the vertebrate neural tube: a sonic hedgehog morphogenregulated transcriptional network. Development 135, 2489-2503.

Dessaud, E., Ribes, V., Balaskas, N., Yang, L. L., Pierani, A., Kicheva, A., Novitch, B. G., Briscoe, J., and Sasai, N. (2010). Dynamic assignment and maintenance of positional identity in the ventral neural tube by the morphogen sonic hedgehog. PLoS Biol. 8, e1000382. doi:10.1371/journal.pbio. 1000382

Dessaud, E., Yang, L. L., Hill, K., Cox, B., Ulloa, F., Ribeiro, A., Mynett, A.,
Novitch, B. G., and Briscoe, J. (2007). Interpretation of the sonic hedgehog morphogen gradient by a temporal adaptation mechanism. Nature 450, 717-720.

Echelard, Y., Epstein, D. J., St-Jacques, B., Shen, L., Mohler, J., McMahon, J. A., and McMahon, A. P. (1993). Sonic hedgehog, a member of a family of putative signaling molecules is implicated in the regulation of CNS and limb polarity. Cell 75, 1417-1430.

Epstein, D. J., McMahon, A. P., and Joyner, A. L. (1999). Regionalization of sonic hedgehog transcription along the anteroposterior axis of the mouse central nervous system is regulated by Hnf3-dependent and -independent mechanisms. Development 126, 281-292.

Ericson, J., Morton, S., Kawakami, A., Roelink, H., and Jessell, T. M. (1996). Two critical periods of sonic hedgehog signaling required for the specification of motor neuron identity. Cell 87, 661-673.

Ericson, J., Rashbass, P., Schedl, A., Brenner-Morton, S., Kawakami, A., van Heyningen, V., Jessell, T. M., and Briscoe, J. (1997). Pax6 controls progenitor cell identity and neuronal fate in response to graded Shh signaling. Cell 90, 169-180.

Fischer, T., Faus-Kessler, T., Welzl, G., Simeone, A., Wurst, W., and Prakash, N. (2011). Fgf15-mediated control of neurogenic and proneural gene expression regulates dorsal midbrain neurogenesis. Dev. Biol. 350, 496-510.

Hashimoto-Torii, K., Motoyama, J., Hui, C. C., Kuroiwa, A., Nakafuku, M., and Shimamura, K. (2003). Differential activities of sonic hedgehog mediated by Gli transcription factors define distinct neuronal subtypes in the dorsal thalamus. Mech. Dev. 120, 1097-1111.

Hébert, J. M., and Fishell, G. (2008). The genetics of early telencephalon patterning: some assembly required. Nat. Rev. Neurosci. 9, 678-685.

Hirata, T., Nakazawa, M., Muraoka, O., Nakayama, R., Suda, Y., and Hibi, M. (2006). Zinc-finger genes $\mathrm{Fez}$ and Fez-like function in the establishment of diencephalon subdivisions. Development 133, 3993-4004.

Horowitz, S. S., Blanchard, J. H., and Morin, L. P. (2004). Intergeniculate leaflet and ventral lateral geniculate nucleus afferent connections: an anatomical substrate for functional input from the vestibulovisuomotor system. J. Comp. Neurol. 474, 227-245.
Ingham, P. W., Nakano, Y., and Seger, C. (2011). Mechanisms and functions of hedgehog signalling across the metazoa. Nat. Rev. Genet. 12, 393-406.

Ishibashi, M., and McMahon, A P. (2002). A sonic hedgehogdependent signaling relay regulates growth of diencephalic and mesencephalic primordia in the early mouse embryo. Development 129 , 4807-4819.

Jeong, J. Y., Einhorn, Z., Mathur, P. Chen, L., Lee, S., Kawakami, K. and Guo, S. (2007). Patterning the zebrafish diencephalon by the conserved zinc-finger protein Fezl. Development 134, 127-136.

Jeong, Y., Dolson, D. K., Waclaw, R. R., Matise, M. P., Sussel, L., Campbell, K., Kaestner, K. H., and Epstein, D. J. (2011). Spatial and temporal requirements for sonic hedgehog in the regulation of thalamic interneuron identity. Development 138, 531-541.

Jeong, Y., El-Jaick, K., Roessler, E., Muenke, M., and Epstein, D. J. (2006). A functional screen for sonic hedgehog regulatory elements across a $1 \mathrm{Mb}$ interval identifies long range ventral forebrain enhancers. Development 133, 761-772.

Jessell, T. M. (2000). Neuronal specification in the spinal cord: inductive signals and transcriptional codes. Nat. Rev. Genet. 1, 20-29.

Jones, E. G. (2007). The Thalamus. Cambridge: Cambridge University Press.

Jones, E. G., and Rubenstein, J. L. (2004). Expression of regulatory genes during differentiation of thalamic nuclei in mouse and monkey. J. Comp. Neurol. 477, 55-80.

Kataoka, A., and Shimogori, T. (2008). Fgf8 controls regional identity in the developing thalamus. Development 135, 2873-2881.

Kiecker, C., and Lumsden, A. (2004). Hedgehog signaling from the ZLI regulates diencephalic regional identity. Nat. Neurosci. 7, 1242-1249.

Lupo, G., Harris, W. A., and Lewis, K. E. (2006). Mechanisms of ventral patterning in the vertebrate nervous system. Nat. Rev. Neurosci. 7, 103-114.

Marigo, V., Davey, R. A., Zuo, Y., Cunningham, J. M., and Tabin, C. J. (1996). Biochemical evidence that patched is the hedgehog receptor. Nature 384, 176-179.

Martinez-Ferre, A., and Martinez, S. (2009). The development of the thalamic motor learning area is regulated by Fgf8 expression. J. Neurosci. 29, 13389-13400.
Matise, M. P., and Wang, H. (2011). Sonic hedgehog signaling in the developing CNS where it has been and where it is going. Curr. Top. Dev. Biol. 97, 75-117.

Miyake, A., Nakayama, Y., Konishi, M., and Itoh, N. (2005). Fgf19 regulated by $\mathrm{Hh}$ signaling is required for zebrafish forebrain development. Dev. Biol. 288, 259-275.

Morin, L. P., and Blanchard, J. H. (2005). Descending projections of the hamster intergeniculate leaflet: relationship to the sleep/arousal and visuomotor systems. J. Comp. Neurol.487, 204-216.

Muhr, J., Andersson, E., Persson, M., Jessell, T. M., and Ericson, J. (2001) Groucho-mediated transcriptional repression establishes progenitor cell pattern and neuronal fate in the ventral neural tube. Cell 104, 861-873.

Nakagawa, Y., and O'Leary, D. D. (2001). Combinatorial expression patterns of LIM-homeodomain and other regulatory genes parcellate developing thalamus. J. Neurosci. 21, 2711-2725.

Peukert, D., Weber, S., Lumsden, A., and Scholpp, S. (2011). Lhx2 and Lhx9 determine neuronal differentiation and compartition in the caudal forebrain by regulating Wnt signaling. PLoS Biol. 9, e1001218. doi:10.1371/journal.pbio.1001218

Puelles, L., and Rubenstein, J. L. (1993). Expression patterns of homeobox and other putative regulatory genes in the embryonic mouse forebrain suggest a neuromeric organization. Trends Neurosci. 16, 472-479.

Puelles, L., and Rubenstein, J. L. (2003). Forebrain gene expression domains and the evolving prosomeric model. Trends Neurosci. 26, 469-476.

Robertson, C. P., Braun, M. M., and Roelink, H. (2004). Sonic hedgehog patterning in chick neural plate is antagonized by a Wnt3-like signal. Dev. Dyn. 229, 510-519.

Roelink, H., Augsburger, A., Heemskerk, J., Korzh, V., Norlin, S., Ruiz i Altaba, A., Tanabe, Y., Placzek, M., Edlund, T., Jessell, T. M., and Dodd, J. (1994). Floor plate and motor neuron induction by vhh-1, a vertebrate homolog of hedgehog expressed by the notochord. Cell 76, 761-775.

Scholpp, S., Delogu, A., Gilthorpe, J., Peukert, D., Schindler, S., and Lumsden, A. (2009). Her6 regulates the neurogenetic gradient and neuronal identity in the thalamus. Proc. Natl. Acad. Sci. U.S.A. 106, 19895-19900.

Scholpp, S., Foucher, I., Staudt, N., Peukert, D., Lumsden, A., and Houart, C. (2007). Otx1l, Otx2 and Irxlb establish and position the ZLI 
in the diencephalon. Development 134, 3167-3176.

Scholpp, S., and Lumsden, A. (2010). Building a bridal chamber: development of the thalamus. Trends Neurosci. 33, 373-380.

Scholpp, S., Wolf, O., Brand, M., and Lumsden, A. (2006). Hedgehog signalling from the zona limitans intrathalamica orchestrates patterning of the zebrafish diencephalon. Development 133, 855-864.

Stamataki, D., Ulloa, F., Tsoni, S. V., Mynett, A., and Briscoe, J. (2005). A gradient of Gli activity mediates graded sonic hedgehog signaling in the neural tube. Genes Dev. 19, 626-641.

Stone, D. M., Hynes, M., Armanini, M., Swanson, T. A., Gu, Q., Johnson, R. L., Scott, M. P., Pennica, D., Goddard, A., Phillips, H., Noll, M., Hooper, J. E., de Sauvage, F., and Rosenthal, A. (1996). The tumour-suppressor gene patched encodes a candidate receptor for sonic hedgehog. Nature 384, 129-134.

Suzuki-Hirano, A., Ogawa, M., Kataoka, A., Yoshida, A. C., Itoh, D., Ueno, M., Blackshaw, S., Shimogori, T. (2011). Dynamic spatiotemporal gene expression in embryonic mouse thalamus. J. Comp. Neurol. 519, 528-543.

Szabó, N. E., Zhao, T., Zhou, X., and Alvarez-Bolado, G. (2009). The role of sonic hedgehog of neural origin in thalamic differentiation in the mouse. J. Neurosci. 29, 2453-2466.

Taipale, J., Cooper, M. K., Maiti, T., Beachy, P. A. (2002). Patched acts catalytically to suppress the activity of smoothened. Nature 418, 892-897.

Vieira, C., Garda, A. L., Shimamura, K., and Martinez, S. (2005). Thalamic development induced by Shh in the chick embryo. Dev. Biol. 284, 351-363.

Vieira, C., and Martinez, S. (2005). Experimental study of MAP kinase phosphatase-3 (Mkp3) expression in the chick neural tube in relation to Fgf8 activity. Brain Res. Brain Res. Rev. 49, 158-166.

Vieira, C., and Martinez, S. (2006). Sonic hedgehog from the basal plate and the zona limitans intrathalamica exhibits differential activity on diencephalic molecular regionalization and nuclear structure. Neuroscience 143, 129-140.

Vue, T. Y., Aaker, J., Taniguchi, A., Kazemzadeh, C., Skidmore, J. M., Martin, D. M., Martin, J. F., Treier,
M., and Nakagawa, Y. (2007). Characterization of progenitor domains in the developing mouse thalamus. J. Comp. Neurol. 505, 73-91.

Vue, T. Y., Bluske, K., Alishahi, A., Yang, L. L., Koyano-Nakagawa, N. Novitch, B., and Nakagawa, Y. (2009). Sonic hedgehog signaling controls thalamic progenitor identity and nuclei specification in mice. J. Neurosci. 29, 4484-4497.

Wilson, C. W., and Chuang, P. T. (2010). Mechanism and evolution of cytosolic hedgehog signal transduction Development 137, 2079-2094.

Yuge, K., Kataoka, A., Yoshida, A. C. Itoh, D., Aggarwal, M., Mori, S. Blackshaw, S., and Shimogori, T. (2011). Region-specific gene expression in early postnatal mouse thalamus. J. Comp. Neurol. 519, 544-561.

Zeltser, L. M. (2005). Shh-dependent formation of the ZLI is opposed by signals from the dorsal diencephalon. Development 132, 2023-2033.

Zhang, X. M., Ramalho-Santos, M., and McMahon, A. P. (2001). Smoothened mutants reveal redundant roles for Shh and Ihh signaling including regulation of $\mathrm{L} / \mathrm{R}$ symmetry by the mouse node. Cell 106 781-792.
Zhou, C. J., Pinson, K. I., and Pleasure, S. J. (2004). Severe defects in dorsal thalamic development in lowdensity lipoprotein receptor-related protein-6 mutants. J. Neurosci. 24 , 7632-7639.

Conflict of Interest Statement: The author declares that the research was conducted in the absence of any commercial or financial relationships that could be construed as a potential conflict of interest.

Received: 01 February 2012; paper pending published: 13 February 2012; accepted: 30 March 2012; published online: 18 April 2012.

Citation: Epstein DJ (2012) Regulation of thalamic development by Sonic hedgehog. Front. Neurosci. 6:57. doi: 10.3389/fnins.2012.00057

This article was submitted to Frontiers in Neurogenesis, a specialty of Frontiers in Neuroscience.

Copyright (c) 2012 Epstein. This is an open-access article distributed under the terms of the Creative Commons Attribution Non Commercial License, which permits non-commercial use, distribution and reproduction in other forums, provided the original authors and source are credited. 